\title{
Flow of an Eyring-Powell Model Fluid between Coaxial Cylinders with Variable Viscosity
}

\author{
Azad Hussain, M. Y. Malik, and Farzana Khan \\ Department of Mathematics, Quaid-i-Azam University, Islamabad 45320, Pakistan \\ Correspondence should be addressed to Azad Hussain; azadhussainsamote@yahoo.com
}

Received 21 July 2013; Accepted 18 August 2013

Academic Editors: G. Chen and S. Wei-dong

Copyright ( 2013 Azad Hussain et al. This is an open access article distributed under the Creative Commons Attribution License, which permits unrestricted use, distribution, and reproduction in any medium, provided the original work is properly cited.

\begin{abstract}
We consider the flow of Eyring-Powell model fluid in the annulus between two cylinders whose viscosity depends upon the temperature. We consider the steady flow in the annulus due to the motion of inner cylinder and constant pressure gradient. In the problem considered the flow is found to be remarkedly different from that for the incompressible Navier-Stokes fluid with constant viscosity. An analytical solution of the nonlinear problem is obtained using homotopy analysis method. The behavior of pertinent parameters is analyzed and depicted through graphs.
\end{abstract}

\section{Introduction}

The analysis of the behaviour of the fluid motion of the non-Newtonian fluids becomes much complicated and subtle as compared to Newtonian fluids due to the fact that nonNewtonian fluids do not exhibit the linear relationship between stress and strain. Rivlin and Ericksen [1] and Truesdell and Noll [2] classified viscoelastic fluids with the help of constitutive relations for the stress tensor as a function of the symmetric part of the velocity gradient and its higher (total) derivatives. In recent years, there have been several studies [3-12] on flows of non-Newtonian fluids. It is a well-known fact that it is not possible to obtain a single constitutive equation exhibiting all properties of all nonNewtonian fluids from the available literature. That is why several models of non-Newtonian fluids have been proposed in the literature. Eyring-Powell model fluid is one of these models. Eyring-Powell model was first introduced by Powell and Eyring in 1944. However, the literature survey indicates that very low energy has been devoted to the flows of EyringPowell model fluid with variable viscosity. Massoudi and Christie [13] have considered the effects of variable viscosity and viscous dissipation on the flow of a third grade fluid in a uniform pipe. Massoudi and Christie [13] found the numerical solutions with the help of straight forward finite difference method. They also discussed that the flow of a fluid-solid mixture is very complicated and may depend on many variables such as physical properties of each phase and size and shape of solid particles. Later on, the influence of constant and space dependent viscosity on the flow of a third grade fluid in a pipe has been discussed analytically by Hayat et al. [14]. The approximate and analytical solution of non-Newtonian fluid with variable viscosity has been analyzed by Yürüsoy and Pakdermirli [15] and Pakdemirli and Yilbas [16]. The pipe flow of non-Newtonian fluid with variable viscosity keeping no slip and partial slip has been discussed analytically by Nadeem and Ali [17] and Nadeem et al. [18]. More recently, Nadeem and Akbar [19] studied the effects of temperature dependent viscosity on peristaltic flow of a Jeffrey-six constant fluid in a uniform vertical tube. The main aim of the present study is to venture further in the regime of Eyring-Powell model fluid with variable viscosity. To the best of the authors knowledge no attempt has been made to investigate Eyring-Powell model fluid in the annulus between two cylinders whose viscosity depends upon the temperature. The governing equations for EyringPowell model fluid are formulated considering cylindrical coordinates system. The equations are simplified using the assumptions of long wave length and low Reynolds number approximation. The obtained non-linear problem is solved 
using homotopy analysis method [20-28]. The effects of the emerging parameters are analyzed and depicted through graphs.

\section{Mathematical Model}

The constitutive equation for a Cauchy stress in an EyringPowell model fluid is given by

$$
\begin{gathered}
\overline{\mathbf{S}}=\mu \nabla \bar{V}+\frac{1}{\beta} \sinh ^{-1}\left(\frac{1}{c} \nabla \bar{V}\right), \\
\sinh ^{-1}\left(\frac{1}{c} \nabla \bar{V}\right) \approx \frac{1}{c} \nabla \bar{V}-\frac{1}{6}\left(\frac{1}{c} \nabla \bar{V}\right)^{3}, \quad\left|\frac{1}{c} \nabla \bar{V}\right| \ll 1,
\end{gathered}
$$

where $\bar{V}$ is the velocity, $\overline{\mathbf{S}}$ is the Cauchy stress tensor, $\mu$ is the coefficient of shear viscosity, and $\beta$ and $c$ are the material constants. We take the velocity and stress as

$$
\mathbf{V}(r)=\left(\begin{array}{l}
0 \\
0 \\
v
\end{array}\right), \quad \mathbf{S}(r)=\left[\begin{array}{lll}
S_{r r} & S_{r \theta} & S_{r z} \\
S_{\theta r} & S_{\theta \theta} & S_{\theta z} \\
S_{z r} & S_{z \theta} & S_{z z}
\end{array}\right] .
$$

\section{Physical Model}

Consider the steady flow of an Eyring-Powell model fluid with variable temperature dependent viscosity between coaxial cylinders. The motion is caused due to a constant pressure gradient and by the motion of the inner cylinder parallel to its length, whereas the outer cylinder is kept stationary. The heat transfer analysis is also taken into account. The dimensionless problem which can describe the flow is

$$
\begin{gathered}
\frac{\mu}{r} \frac{d v}{d r}+\frac{M}{r} \frac{d v}{d r}+\mu \frac{d^{2} v}{d r^{2}}+M \frac{d^{2} v}{d r^{2}} \\
-3 K\left(\frac{d v}{d r}\right)^{2} \frac{d^{2} v}{d r^{2}}-\frac{K}{r}\left(\frac{d v}{d r}\right)^{3}-B=0 \\
\mu \Gamma\left(\frac{d v}{d r}\right)^{2}+M \Gamma\left(\frac{d v}{d r}\right)^{2}-\Gamma K\left(\frac{d v}{d r}\right)^{4}-\frac{1}{r} \frac{d \theta}{d r}-\frac{d^{2} \theta}{d r^{2}}=0 \\
v(r)=1, \quad \theta(r)=1, \quad r=1 \\
v(r)=0, \quad \theta(r)=0, \quad r=b
\end{gathered}
$$

whence

$$
\begin{aligned}
& r=\frac{\bar{r}}{R}, \quad \Gamma=\frac{\mu_{*} V_{0}^{2}}{k\left(\theta_{m}-\theta_{w}\right)}, \quad \theta=\frac{\left(\bar{\theta}-\theta_{w}\right)}{\left(\theta_{m}-\theta_{w}\right)}, \\
& z=\frac{V_{0}^{2}}{R^{2} c^{2}}, \quad C_{1}=\frac{\partial p}{\partial z}, \quad M=\frac{1}{\beta c \mu_{*}}, \\
& K=\frac{V_{0}^{2}}{R^{2} c^{2}}, \quad B=\frac{C_{1} R^{2}}{\mu_{*} V_{0}}, \quad v=\frac{\bar{v}}{v_{0}}, \quad \mu=\frac{\bar{\mu}}{\mu_{*}},
\end{aligned}
$$

where $\mu_{*}, \theta_{m}, V_{0}$ and $\Gamma$ are, respectively, the reference viscosity, a reference temperature (the bulk mean fluid temperature), and reference velocity $\Gamma$ is related to the Prandtl number and Eckert number.

\section{Series Solutions for Reynolds' Model}

Here the viscosity is expressed in the form

$$
\mu=e^{-P \theta}
$$

which by Maclaurin's series can be written as

$$
\mu=1-P \theta+O\left(\theta^{2}\right)
$$

Note that $M=0$ corresponds to the case of constant viscosity. Invoking the above equation into (3) one has

$$
\begin{aligned}
& \frac{M}{r} \frac{d v}{d r}+\frac{1}{r} \frac{d v}{d r}-\frac{P \theta}{r} \frac{d v}{d r}+\frac{d^{2} v}{d r^{2}}-P \theta \frac{d^{2} v}{d r^{2}} \\
& +M \frac{d^{2} v}{d r^{2}}-3 K\left(\frac{d v}{d r}\right)^{2} \frac{d^{2} v}{d r^{2}}-\frac{K}{r}\left(\frac{d v}{d r}\right)^{3}-B=0, \\
& \Gamma\left(\frac{d v}{d r}\right)^{2}-\Gamma P \theta\left(\frac{d v}{d r}\right)^{2}+M \Gamma\left(\frac{d v}{d r}\right)^{2} \\
& -\Gamma K\left(\frac{d v}{d r}\right)^{4}-\frac{1}{r} \frac{d \theta}{d r}-\frac{d^{2} \theta}{d r^{2}}=0 .
\end{aligned}
$$

For HAM solution, we choose the following initial guesses:

$$
v_{0}(r)=\frac{(r-b)}{(1-b)}, \quad \theta_{0}(r)=\frac{(r-b)}{(1-b)} .
$$

The auxiliary linear operators are in the form

$$
\begin{aligned}
& \mathfrak{E}_{v r}(v)=v^{\prime \prime}, \\
& \mathfrak{E}_{\theta r}(\theta)=\theta^{\prime \prime}
\end{aligned}
$$

which satisfy

$$
\mathscr{L}_{v r}\left(A_{1}+B_{1} r\right)=0, \quad \mathscr{L}_{\theta r}\left(A_{2}+B_{2} r\right)=0,
$$

where $A_{1}, A_{2}, B_{1}$, and $B_{2}$ are the constants.

If $p \in[0,1]$ is an embedding parameter and $h_{v}$ and $h_{\theta}$ are auxiliary parameters, then the problems at the zero and $m$ th order are, respectively, given by

$$
(1-p) \mathscr{L}_{v}\left[\bar{v}(r, p)-v_{0}(r)\right]=p \hbar_{v} N_{v}[\bar{v}(r, p), \bar{\theta}(r, p)],
$$

$$
(1-p) \mathscr{L}_{\theta}\left[\bar{\theta}(r, p)-\theta_{0}(r)\right]=p \hbar_{\theta} N_{\theta}[\bar{v}(r, p), \bar{\theta}(r, p)]
$$

$$
\begin{gathered}
\mathscr{L}_{v}\left[v_{m}(r)-\chi_{m} v_{m-1}(r)\right]=\hbar_{v} R_{v}(r), \\
\mathscr{L}_{\theta}\left[\theta_{m}(r)-\chi_{m} \theta_{m-1}(r)\right]=\hbar_{\theta} R_{\theta}(r), \\
\bar{v}(r, p)=\bar{\theta}(r, p)=1, \quad r=1, \\
\bar{v}(r, p)=\bar{\theta}(r, p)=0, \quad r=b .
\end{gathered}
$$

The boundary conditions at the $m$ th order are

$$
\begin{array}{ll}
\bar{v}_{m}(r, p)=\bar{\theta}_{m}(r, p)=0, & r=1, \\
\bar{v}_{m}(r, p)=\bar{\theta}_{m}(r, p)=0, & r=b .
\end{array}
$$


In (11)-(13)

$$
\begin{aligned}
& N_{v}[\bar{v}(r, p), \bar{\theta}(r, p)] \\
&=-\frac{P \theta}{r} \frac{d v}{d r}+\frac{1}{r} \frac{d v}{d r}+\frac{M}{r} \frac{d v}{d r}+\frac{d^{2} v}{d r^{2}}-P \theta \frac{d^{2} v}{d r^{2}} \\
&+M \frac{d^{2} v}{d r^{2}}-3 K\left(\frac{d v}{d r}\right)^{2} \frac{d^{2} v}{d r^{2}}-\frac{K}{r}\left(\frac{d v}{d r}\right)^{3}-B \\
& N_{\theta}[\bar{v}(r, p), \bar{\theta}(r, p)] \\
&=\Gamma\left(\frac{d v}{d r}\right)^{2}-\Gamma P \theta\left(\frac{d v}{d r}\right)^{2} \\
&+M \Gamma\left(\frac{d v}{d r}\right)^{2}-\Gamma K\left(\frac{d v}{d r}\right)^{4}-\frac{1}{r} \frac{d \theta}{d r}-\frac{d^{2} \theta}{d r^{2}} \\
& R_{v}=-\frac{P}{r} \sum_{k=0}^{m-1} v_{m-1-k}^{\prime} \theta_{k}+\frac{1}{r} v_{m-1}^{\prime}+\frac{M}{r} v_{m-1}^{\prime} \\
&+v_{m-1}^{\prime \prime}-P \sum_{k=0}^{m-1} v_{m-1-k}^{\prime \prime} \theta_{k} \\
&+M v_{m-1}^{\prime \prime}-3 K \sum_{k=0}^{m-1} \sum_{l=0}^{k} v_{m-1-k}^{\prime} v_{k-l}^{\prime} v_{l}^{\prime \prime} \\
&-\Gamma K \sum_{k=0}^{m-1} \sum_{l=0}^{k} \sum_{s=0}^{l} v_{m-1-k}^{\prime} v_{k-l}^{\prime} v_{l-s}^{\prime} v_{s}^{\prime}-\frac{1}{r} \theta_{m-1}^{\prime}-\theta_{m-1}^{\prime \prime} \\
&-\frac{K}{r} \sum_{k=0}^{m-1} \sum_{l=0}^{k} v_{m-1-k}^{\prime} v_{k-l}^{\prime} v_{l}^{\prime}-B, \\
& R_{\theta}= \Gamma \sum_{k=0}^{m-1} v_{m-1-k}^{\prime} v_{k}^{\prime}-\Gamma P \sum_{k=0}^{m-1} \sum_{l=0}^{m} v_{m-1-k}^{\prime} v_{k-l}^{\prime} \theta_{l}^{\prime} \\
& M \Gamma v_{m-1-k}^{\prime} v_{k}^{\prime}
\end{aligned}
$$

By Mathematica the solutions of (21) can be written as

$$
\begin{aligned}
v_{m}(r) & =\sum_{n=0}^{3 m} a_{m, n} r^{n}, \quad m \geq 0, \\
\theta_{m}(r) & =\sum_{n=0}^{3 m+1} d_{m, n} r^{n}, \quad m \geq 0,
\end{aligned}
$$

where $a_{m, n}$ and $d_{m, n}$ are constants which can be determined on substituting (22) into (15) and (16).

\section{Series Solutions for Vogel's Model}

Here

$$
\mu=\mu_{*} \exp \left[\frac{A}{(t+\theta)}-\theta_{w}\right]
$$

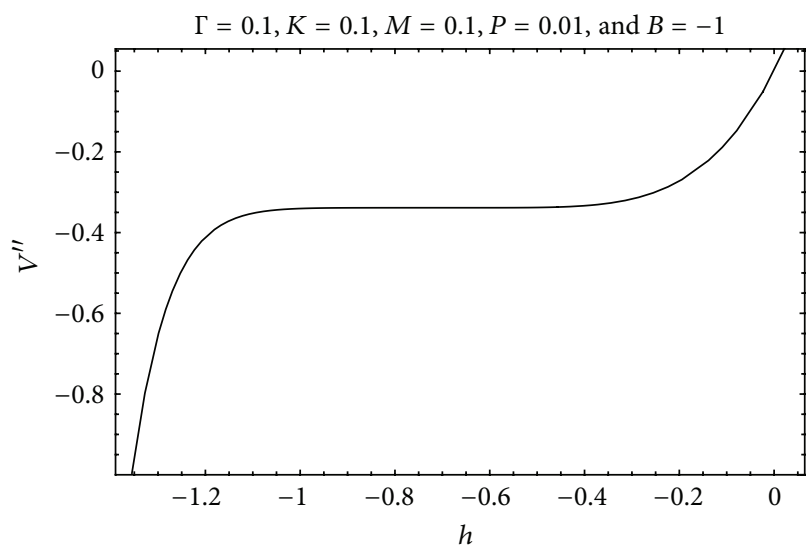

FIGURE 1: $h$-curve for Reynolds' model for velocity profile.

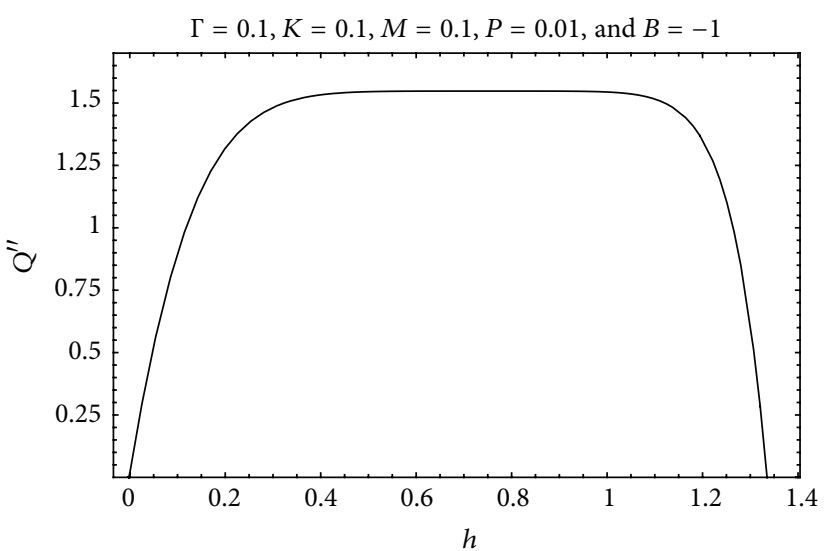

FIGURE 2: $h$-curve for Reynolds' model for temperature profile.

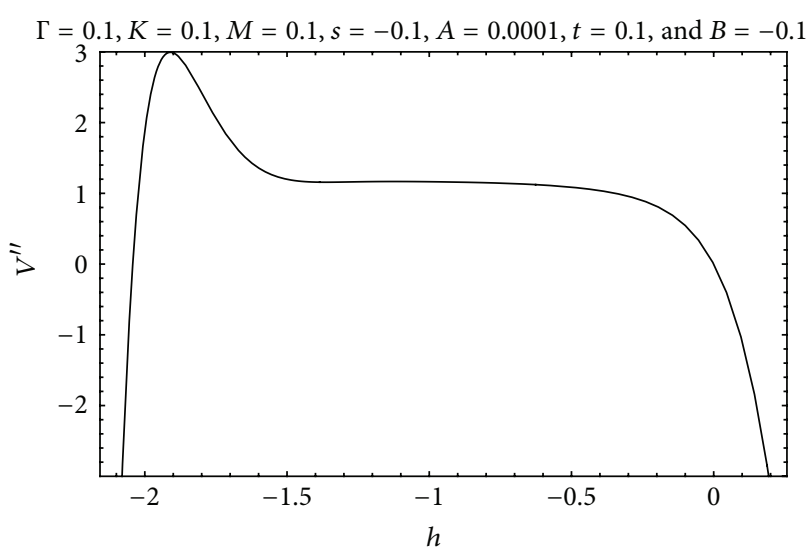

Figure 3: $h$-curve for Vogel's model for velocity profile.

which by Maclaurin's series reduces to

$$
\mu=\frac{B}{s}\left(1-\frac{A \theta}{t^{2}}\right)
$$




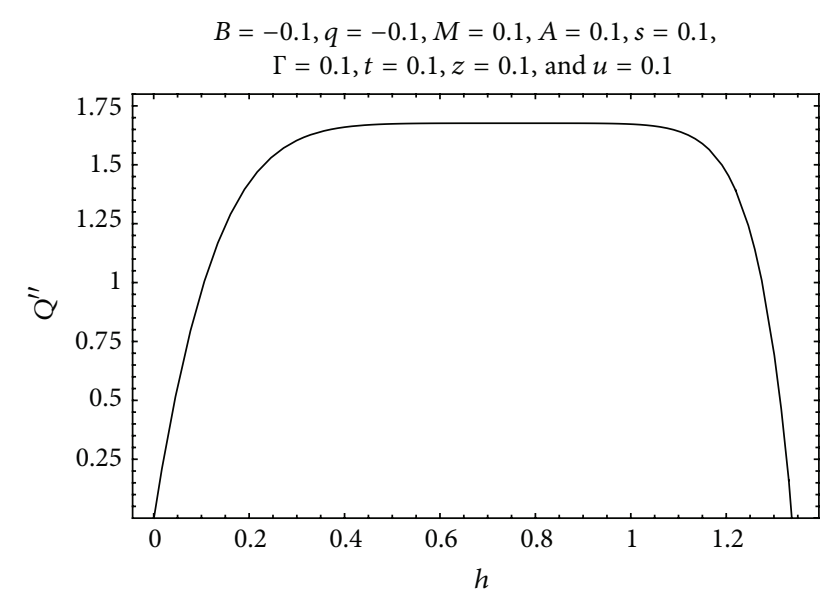

FIGURE 4: $h$-curve for Vogel's model for temperature profile.

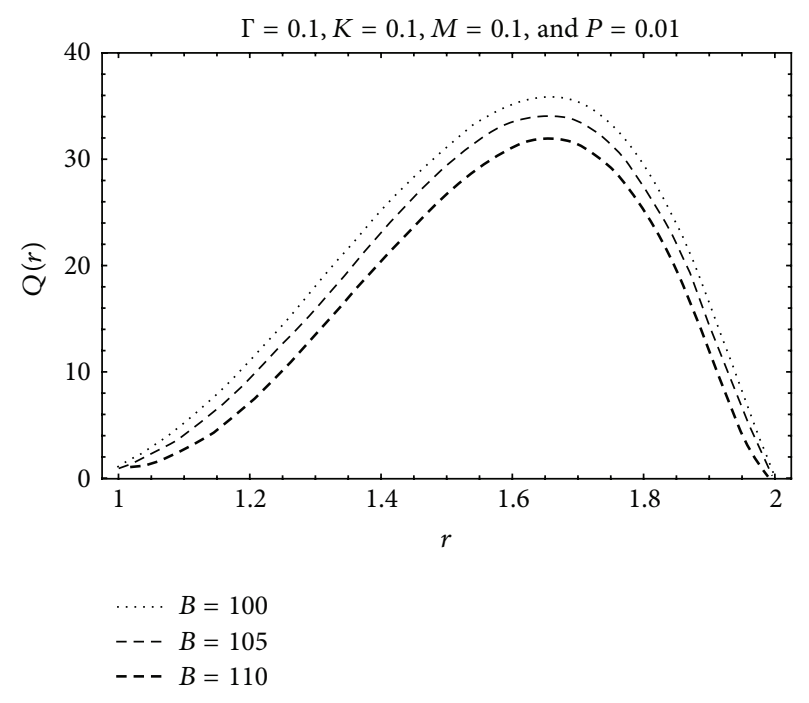

FIGURE 5: Temperature profile for Reynolds' model for different values of $B$.

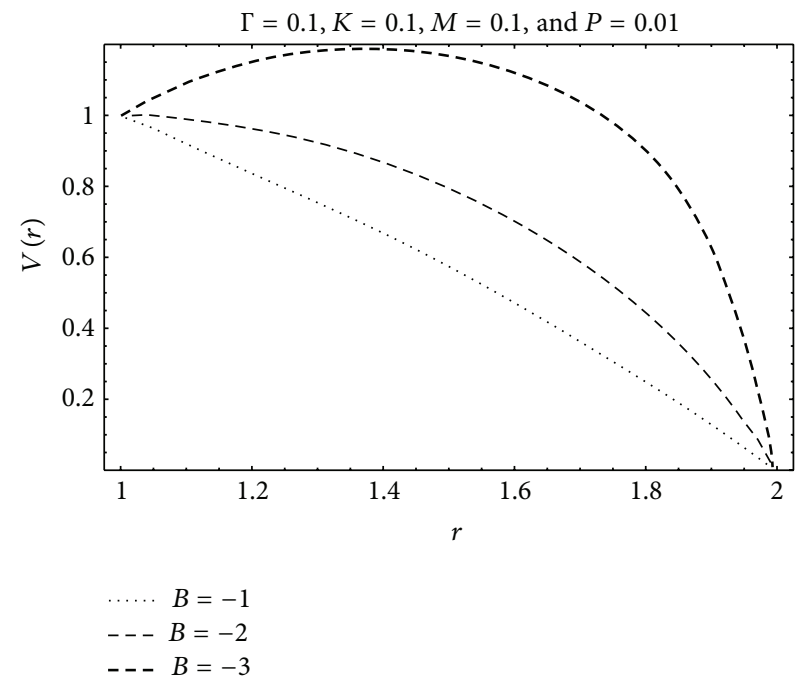

FIGURE 6: Velocity profile for Reynolds' model for different values of $B$.

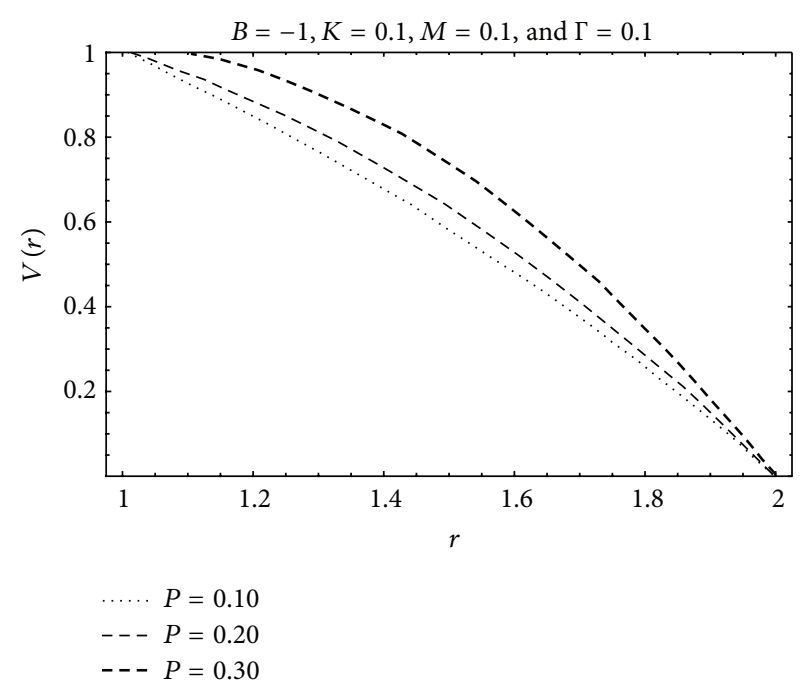

FIGURE 7: Velocity profile for Reynolds' model for different values of $P$.

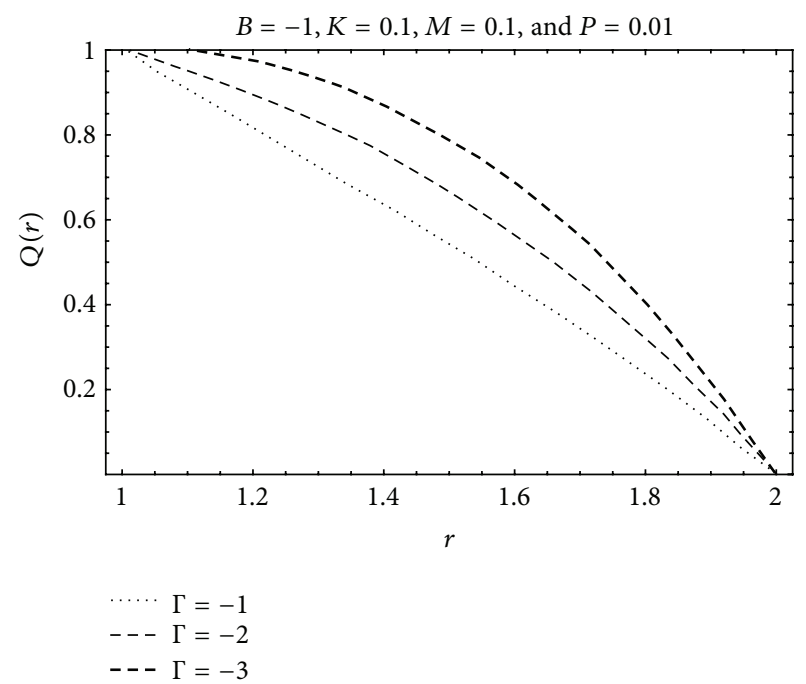

FIGURE 8: Temperature profile for Reynolds' model for different values of $\Gamma$.

Invoking the above expressions, (1) become

$$
\begin{gathered}
-\frac{A B \theta}{r s t^{2}} \frac{d v}{d r}+\frac{1}{r} \frac{d v}{d r}+\frac{M}{r} \frac{d v}{d r}+\frac{B}{s} \frac{d^{2} v}{d r^{2}}-\frac{A B}{s t^{2}} \theta \frac{d^{2} v}{d r^{2}} \\
+M \frac{d^{2} v}{d r^{2}}-3 K\left(\frac{d v}{d r}\right)^{2} \frac{d^{2} v}{d r^{2}}-\frac{K}{r}\left(\frac{d v}{d r}\right)^{3}-B=0 \\
\Gamma\left(\frac{d v}{d r}\right)^{2}-\Gamma \frac{A B}{s t^{2}} \theta\left(\frac{d v}{d r}\right)^{2}+M \Gamma\left(\frac{d v}{d r}\right)^{2} \\
-\Gamma K\left(\frac{d v}{d r}\right)^{4}-\frac{1}{r} \frac{d \theta}{d r}-\frac{d^{2} \theta}{d r^{2}}=0 .
\end{gathered}
$$




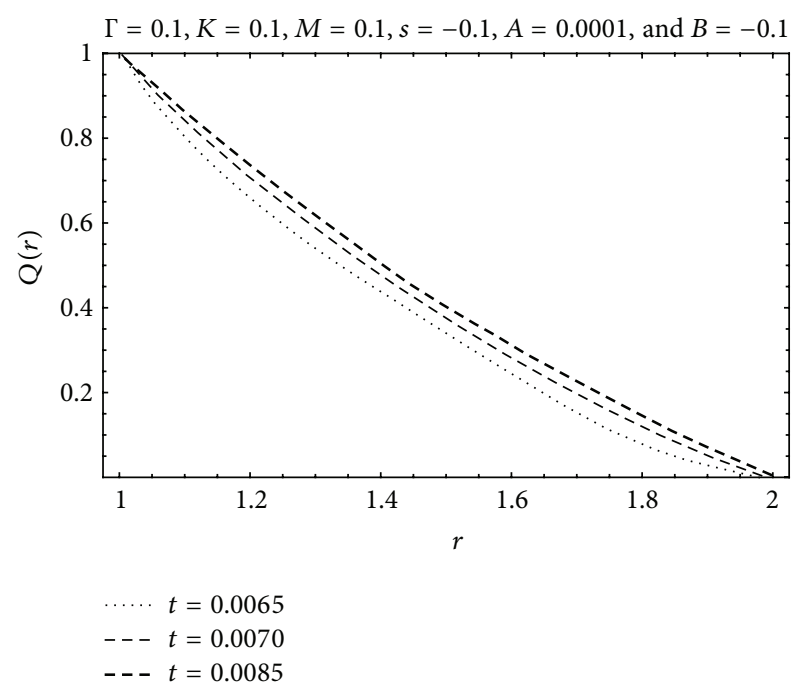

Figure 9: Temperature profile for Vogel's model for different values of $t$.

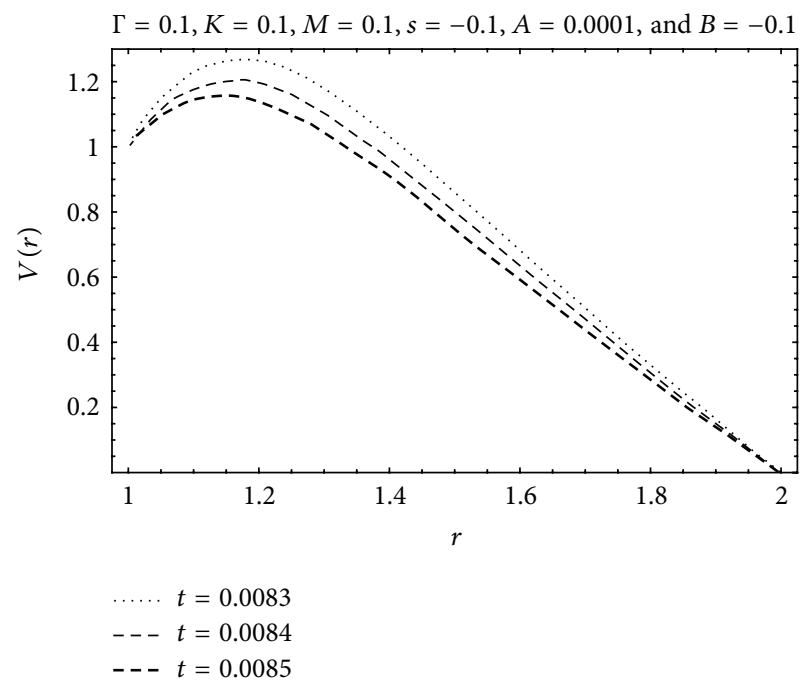

FIGURE 10: Velocity profile for Vogel's model for different values of $t$.

With the following initial guesses and auxiliary linear operators

$$
\begin{aligned}
v_{0 v}(r)=\frac{(r-b)}{(1-b)}, & \theta_{0 v}(r)=\frac{(r-b)}{(1-b),} \\
\mathfrak{E}_{v v}(v)=v^{\prime \prime}, & \mathfrak{E}_{\theta v}(\theta)=\theta^{\prime \prime},
\end{aligned}
$$

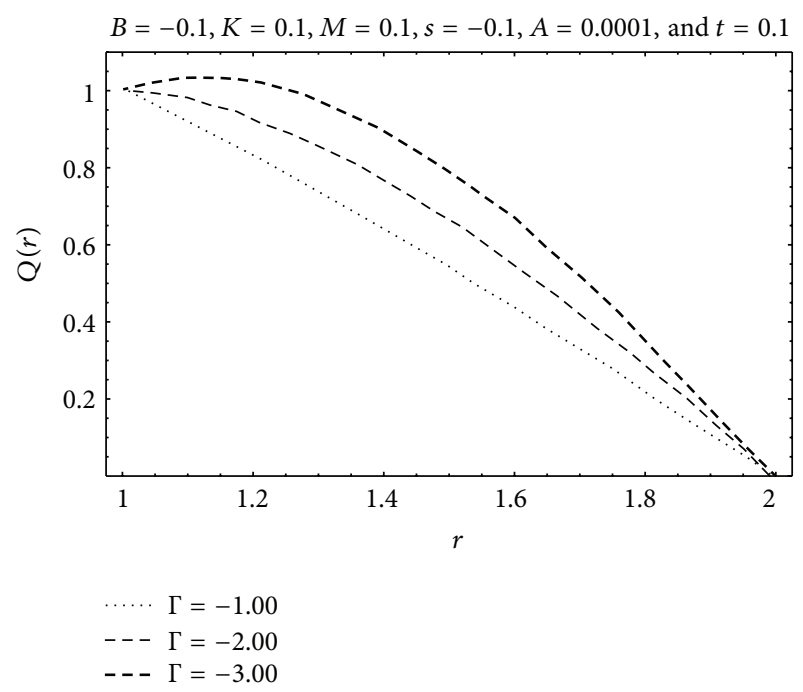

FIGURE 11: Temperature profile for Vogel's model for different values of $\Gamma$.

the $m$ th-order deformation problems are

$$
\begin{gathered}
\mathfrak{E}_{v r}\left[v_{m}(r)-\chi_{m} v_{m-1}(r)\right]=\hbar_{v} R_{v r}(r), \\
\mathfrak{E}_{\theta r}\left[\theta_{m}(r)-\chi_{m} \theta_{m-1}(r)\right]=\hbar_{\theta} R_{\theta r}(r), \\
N_{v}[\bar{v}(r, p), \bar{\theta}(r, p)] \\
=-\frac{A B \theta}{r s t^{2}} \frac{d v}{d r}+\frac{1}{r} \frac{d v}{d r}+\frac{M}{r} \frac{d v}{d r}+\frac{B}{s} \frac{d^{2} v}{d r^{2}}+M \frac{d^{2} v}{d r^{2}} \\
-\frac{A B}{s t^{2}} \theta \frac{d^{2} v}{d r^{2}}-3 K\left(\frac{d v}{d r}\right)^{2} \frac{d^{2} v}{d r^{2}}-\frac{K}{r}\left(\frac{d v}{d r}\right)^{3}-B, \\
N_{\theta}[\bar{v}(r, p), \bar{\theta}(r, p)] \\
=\Gamma\left(\frac{d v}{d r}\right)^{2}-\Gamma \frac{A B}{s t^{2}} \theta\left(\frac{d v}{d r}\right)^{2}+M \Gamma\left(\frac{d v}{d r}\right)^{2} \\
-\Gamma K\left(\frac{d v}{d r}\right)^{4}-\frac{1}{r} \frac{d \theta}{d r}-\frac{d^{2} \theta}{d r^{2}}, \\
N_{v}[\bar{v}(r, p), \bar{\theta}(r, p)] \\
=-\frac{A B}{r s t^{2}} \sum_{k=0}^{m-1} v_{m-1-k}^{\prime} \theta_{k}+\frac{1}{r} v_{m-1}^{\prime} \\
+\frac{M}{r} v_{m-1}^{\prime}+\frac{B}{s} v_{m-1}^{\prime \prime} \\
-\frac{A B}{s t^{2}} \sum_{k=0}^{m-1} v_{m-1-k}^{\prime \prime} \theta_{k}+M v_{m-1}^{\prime \prime} \\
-3 K \sum_{k=0}^{m-1} \sum_{l=0}^{k} v_{m-1-k}^{\prime} v_{k-l}^{\prime} v_{l}^{\prime \prime} \\
{ }^{\prime \prime}
\end{gathered}
$$




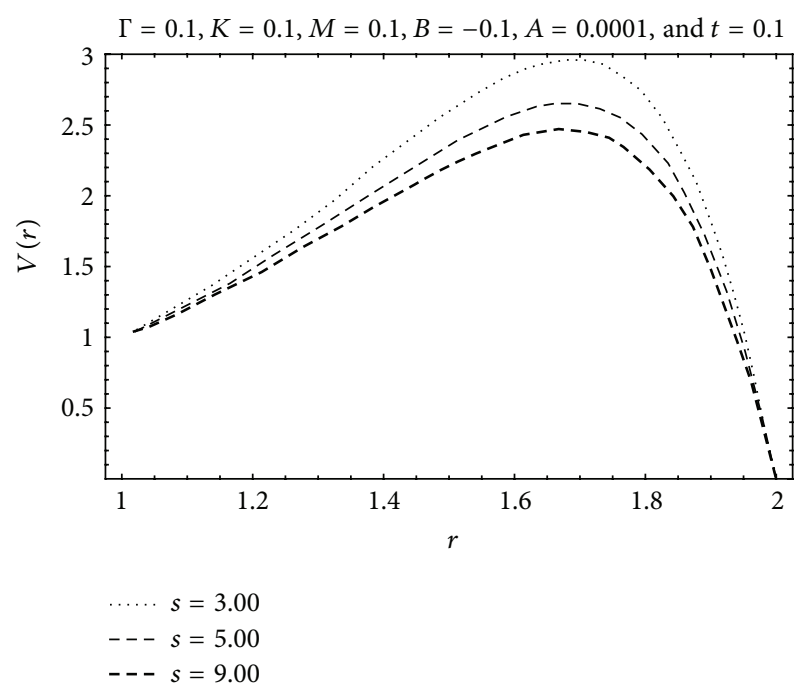

FigURE 12: Velocity profile for Vogel's model for different values of $s$.

$$
\begin{aligned}
& -\frac{K}{r} \sum_{k=0}^{m-1} \sum_{l=0}^{k} v_{m-1-k}^{\prime} v_{k-l}^{\prime} v_{l}^{\prime}-B, \\
& N_{\theta}[\bar{v}(r, p), \bar{\theta}(r, p)] \\
& =\Gamma \sum_{k=0}^{m-1} v_{m-1-k}^{\prime \prime} v_{k}^{\prime} \\
& -\Gamma \frac{A B}{s t^{2}} \sum_{k=0}^{m-1} \sum_{l=0}^{k} v_{m-1-k}^{\prime} v_{k-l}^{\prime} \theta_{l}^{\prime} \\
& +M \Gamma \sum_{k=0}^{m-1} v_{m-1-k}^{\prime} v_{k}^{\prime} \\
& -\Gamma K \sum_{k=0}^{m-1} \sum_{l=0}^{k} \sum_{s=0}^{l} v_{m-1-k}^{\prime} v_{k-l}^{\prime} v_{l-s}^{\prime} v_{s}^{\prime} \\
& -\frac{1}{r} \theta_{m-1}^{\prime}-\theta_{m-1}^{\prime \prime} .
\end{aligned}
$$

The expressions of $v_{m}$ and $\theta_{m}$ are finally given by

$$
\begin{gathered}
v_{m}(r)=\sum_{n=0}^{3 m} a_{m, n}^{\prime} r^{n}, \quad m \geq 0, \\
\theta_{m}(r)=\sum_{n=0}^{3 m+1} d_{m, n}^{\prime} r^{n}, \quad m \geq 0 .
\end{gathered}
$$

\section{Graphical Results and Discussion}

In order to report the convergence of the obtained series solutions and the effects of sundry parameters in the present

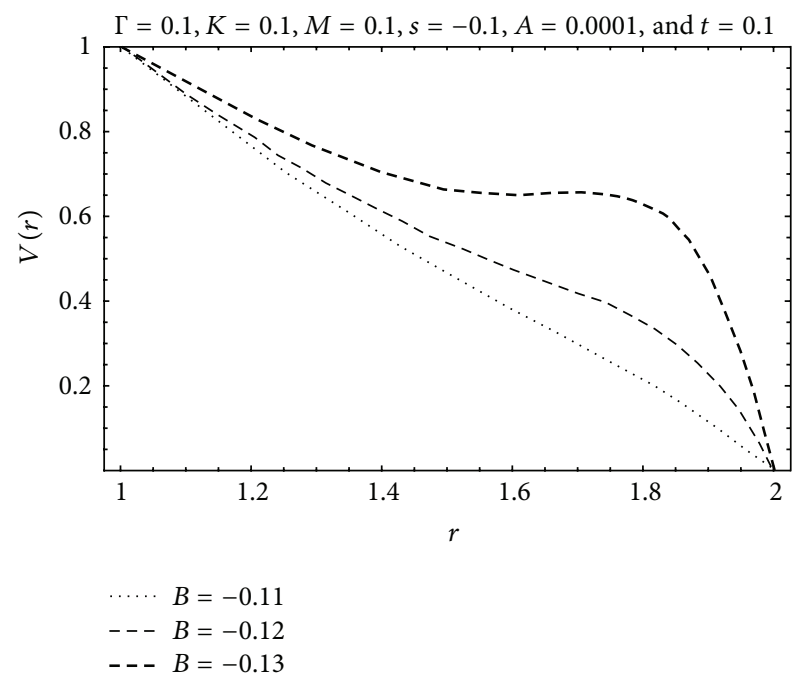

FIGURE 13: Velocity profile for Vogel's model for different values of $B$.

investigation we plotted Figures 1-13. Figures 1-4 are prepared to see the convergence region. Figures 1 and 2 correspond to Reynolds' model whereas Figures 3 and 4 relate to Vogel's model. Figure 5 shows the temperature variation for different values of $B$ for Reynolds' model. It can be seen that temperature decreases as $B$ increases. Figure 6 depicts the velocity variation for Reynolds' model for different values of $B$. Velocity also decreases as $B$ increases. Figure 7 shows the velocity variation for different values of $P$ for Reynolds' model. It can be seen that velocity increases as $P$ increases. Figure 8 is plotted in order to see the temperature variation for Reynolds' model for different values of $\Gamma$; it is depicted that temperature increases as $\Gamma$ increases. Figures $9-13$ correspond to Vogel's model. Figure 9 depicts temperature variation for Vogel's model for different values of $t$. It is seen that temperature increases as $t$ increases. Figure 10 shows the velocity variation for Vogel's model for different values of $t$. It is observed that velocity decreases as $t$ increases. Figure 11 is prepared to observe the temperature variation for Vogel's model for different values of $\Gamma$. It is observed that temperature decreases as $\Gamma$ increases. Figure 12 is plotted to see the the velocity variation for Vogel's model for different values of $s$. It is observed that velocity decreases as $s$ increases. Figure 13 depicts the velocity variation for Vogel's model for different values of $B$. It is observed that velocity decreases as $B$ increases.

\section{Conclusions}

In this paper, we consider the flow of Eyring-Powell model fluid in the annulus between two cylinders whose viscosity depends upon the temperature. We discussed the steady flow in the annulus due to the motion of inner cylinder and constant pressure gradient. In the problem considered the flow is found to be remarkedly different from that for the incompressible Navier-Stokes fluid with constant viscosity. The behavior of pertinent parameters is analyzed 
and depicted through graphs. Using usual similarity transformations the governing equations have been transformed into non-linear ordinary differential equations. The highly nonlinear problem is then solved by homotopy analysis method. Effects of the various parameters on velocity and temperature profiles are examined.

\section{Conflict of Interests}

The authors declare that there is no conflict of interests regarding the publication of this paper.

\section{References}

[1] R. S. Rivlin and J. L. Ericksen, "Stress deformation relations for isotropic materials," Rational Mechanics and Analysis Journal, vol. 4, pp. 323-425, 1955.

[2] C. Truesdell and W. Noll, The Non-Linear Field Theories of Mechanics, Springer, New York, NY, USA, 2nd edition, 1992.

[3] K. R. Rajagopal, "A note on unsteady unidirectional flows of a non-Newtonian fluid," International Journal of Non-Linear Mechanics, vol. 17, no. 5-6, pp. 369-373, 1982.

[4] K. R. Rajagopal and A. S. Gupta, "An exact solution for the flow of a non-newtonian fluid past an infinite porous plate," Meccanica, vol. 19, no. 2, pp. 158-160, 1984.

[5] K. R. Rajagopal and R. K. Bhatnagar, "Exact solutions for some simple flows of an Oldroyd-B fluid," Acta Mechanica, vol. 113, no. 1-4, pp. 233-239, 1995.

[6] K. R. Rajagopal, "On the creeping flow of the second-order fluid," Journal of Non-Newtonian Fluid Mechanics, vol. 15, no. 2, pp. 239-246, 1984.

[7] K. R. Rajagopal, "Longitudinal and torsional oscillations of a rod in a non-Newtonian fluid," Acta Mechanica, vol. 49, no. 3-4, pp. 281-285, 1983.

[8] A. M. Benharbit and A. M. Siddiqui, "Certain solutions of the equations of the planar motion of a second grade fluid for steady and unsteady cases," Acta Mechanica, vol. 94, no. 1-2, pp. 85-96, 1992.

[9] T. Hayat, S. Asghar, and A. M. Siddiqui, "Periodic unsteady flows of a non-Newtonian fluid," Acta Mechanica, vol. 131, no. 3-4, pp. 169-175, 1998.

[10] A. M. Siddiqui, T. Hayat, and S. Asghar, "Periodic flows of a non-Newtonian fluid between two parallel plates," International Journal of Non-Linear Mechanics, vol. 34, no. 5, pp. 895-899, 1999.

[11] T. Hayat, S. Asghar, and A. M. Siddiqui, "On the moment of a plane disk in a non-Newtonian fluid," Acta Mechanica, vol. 136, no. 3, pp. 125-131, 1999.

[12] T. Hayat, S. Asghar, and A. M. Siddiqui, "Some unsteady unidirectional flows of a non-Newtonian fluid," International Journal of Engineering Science, vol. 38, no. 3, pp. 337-346, 2000.

[13] M. Massoudi and I. Christie, "Effects of variable viscosity and viscous dissipation on the flow of a third grade fluid in a pipe," International Journal of Non-Linear Mechanics, vol. 30, no. 5, pp. 687-699, 1995.

[14] T. Hayat, R. Ellahi, and S. Asghar, "The influence of variable viscosity and viscous dissipation on the non-Newtonian flow: an analytical solution," Communications in Nonlinear Science and Numerical Simulation, vol. 12, no. 3, pp. 300-313, 2007.
[15] M. Yürüsoy and M. Pakdermirli, "Approximate analytical solutions for flow of a third grade fluid in a pipe," International Journal of Non-Linear Mechanics, vol. 37, no. 2, pp. 187-195, 2002.

[16] M. Pakdemirli and B. S. Yilbas, "Entropy generation for pipe flow of a third grade fluid with Vogel model viscosity," International Journal of Non-Linear Mechanics, vol. 41, no. 3, pp. 432437, 2006.

[17] S. Nadeem and M. Ali, "Analytical solutions for pipe flow of a fourth grade fluid with Reynold and Vogel's models of viscosities," Communications in Nonlinear Science and Numerical Simulation, vol. 14, no. 5, pp. 2073-2090, 2009.

[18] S. Nadeem, T. Hayat, S. Abbasbandy, and M. Ali, "Effects of partial slip on a fourth-grade fluid with variable viscosity: an analytic solution," Nonlinear Analysis: Real World Applications, vol. 11, no. 2, pp. 856-868, 2010.

[19] S. Nadeem and N. S. Akbar, "Effects of temperature dependent viscosity on peristaltic flow of a Jeffrey-six constant fluid in a non-uniform vertical tube," Communications in Nonlinear Science and Numerical Simulation, vol. 15, no. 12, pp. 3950-3964, 2010.

[20] M. Y. Malik, A. Hussain, and S. Nadeem, "Analytical treatment of an oldroyd 8-constant fluid between coaxial cylinders with variable viscosity," Communications in Theoretical Physics, vol. 56, no. 5, pp. 933-938, 2011.

[21] S. Nadeem, T. Hayat, S. Abbasbandy, and M. Ali, "Effects of partial slip on a fourth-grade fluid with variable viscosity: an analytic solution," Nonlinear Analysis: Real World Applications, vol. 11, no. 2, pp. 856-868, 2010.

[22] M. Y. Malik, A. Hussain, S. Nadeem, and T. Hayat, "Flow of a third grade fluid between coaxial cylinders with variable viscosity," Zeitschrift für Naturforschung A, vol. 64, no. 9-10, pp. 588-596, 2009.

[23] S. J. Liao, Beyond Perturbation: Introduction to Homotopy Analysis Method, CRC Press, Boca Raton, Fla, USA, 2003.

[24] S. J. Liao, "On the homotopy analysis method for nonlinear problems," Applied Mathematics and Computation, vol. 147, no. 2, pp. 499-513, 2004.

[25] S. J. Liao, "An analytic solution of unsteady boundary-layer flows caused by an impulsively stretching plate," Communications in Nonlinear Science and Numerical Simulation, vol. 11, no. 3, pp. 326-339, 2006.

[26] S. Abbasbandy, "The application of homotopy analysis method to nonlinear equations arising in heat transfer," Physics Letters A, vol. 360, no. 1, pp. 109-113, 2006.

[27] S. Abbasbandy, "Homotopy analysis method for heat radiation equations," International Communications in Heat and Mass Transfer, vol. 34, no. 3, pp. 380-387, 2007.

[28] S. Abbasbandy, Y. Tan, and S. J. Liao, "Newton-homotopy analysis method for nonlinear equations," Applied Mathematics and Computation, vol. 188, no. 2, pp. 1794-1800, 2007. 

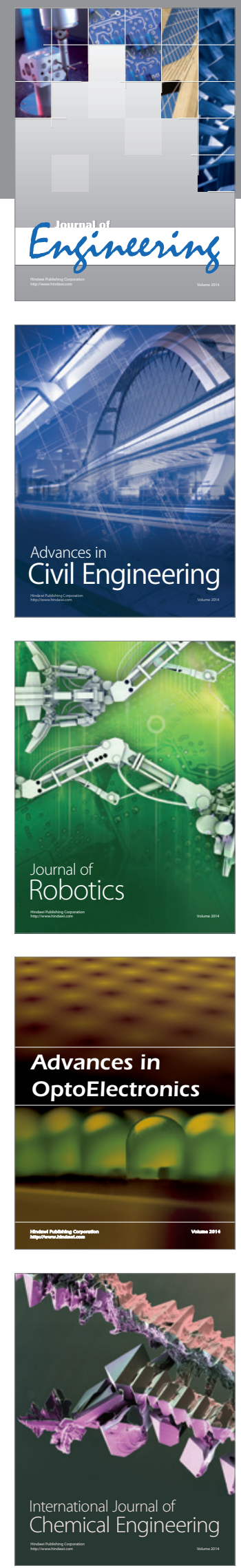

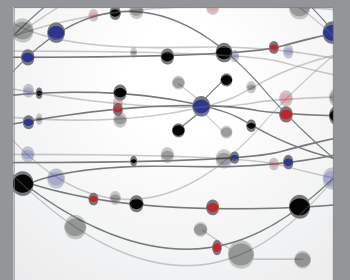

The Scientific World Journal
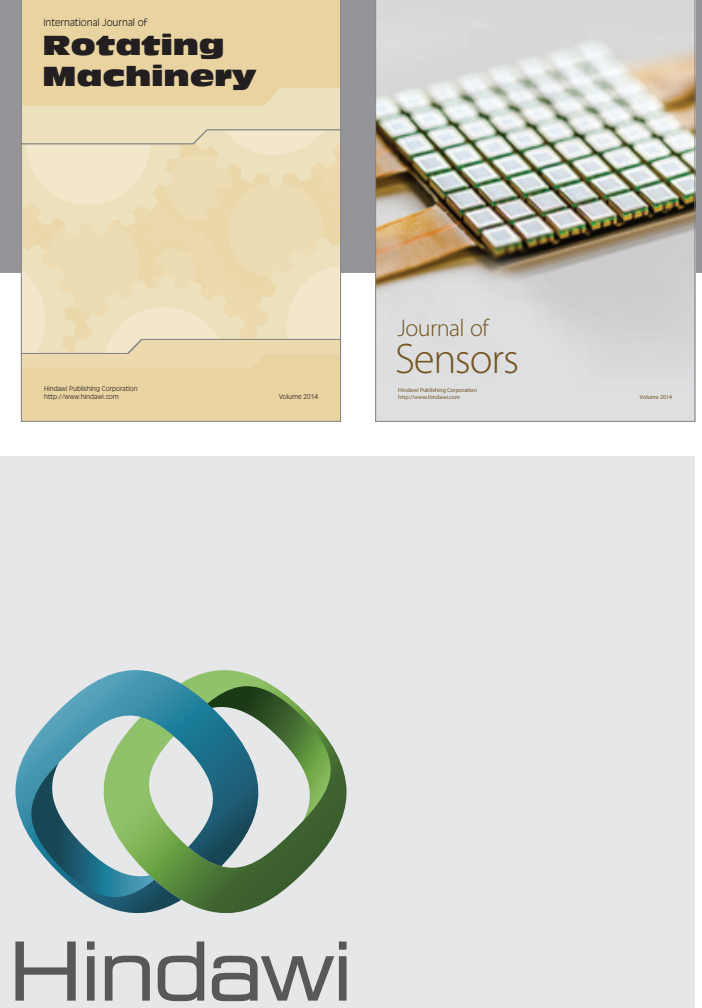

Submit your manuscripts at http://www.hindawi.com
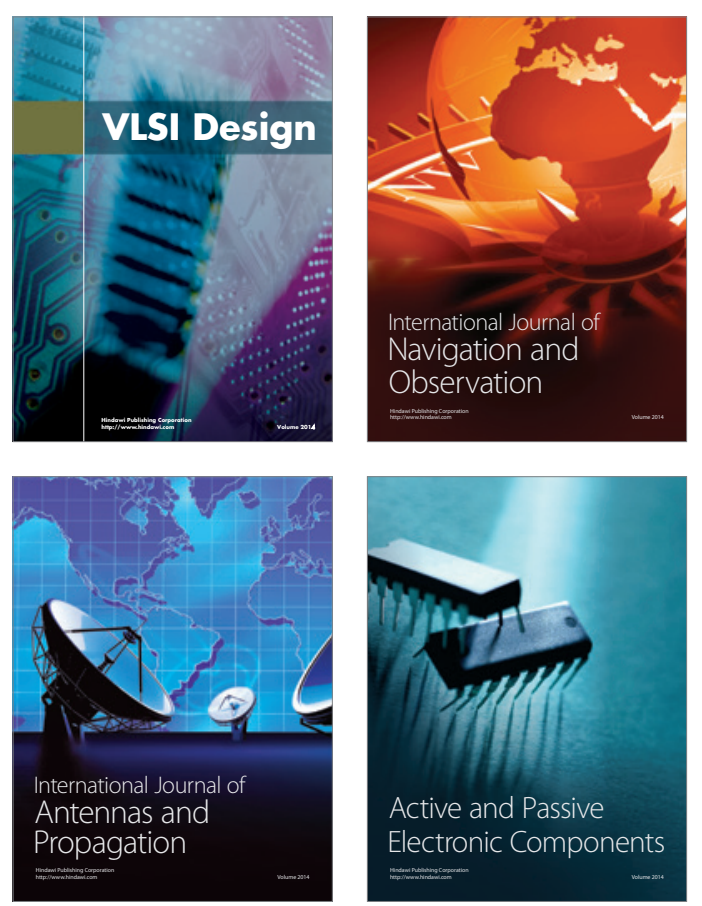
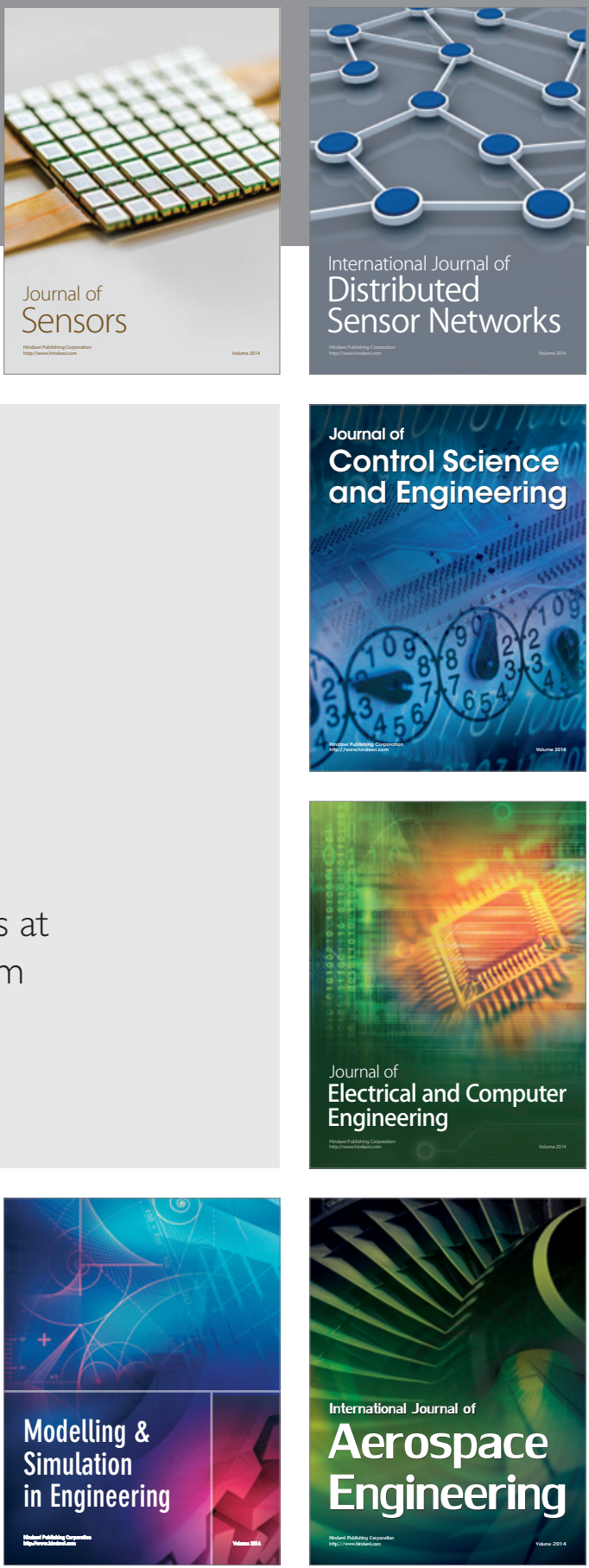

Journal of

Control Science

and Engineering
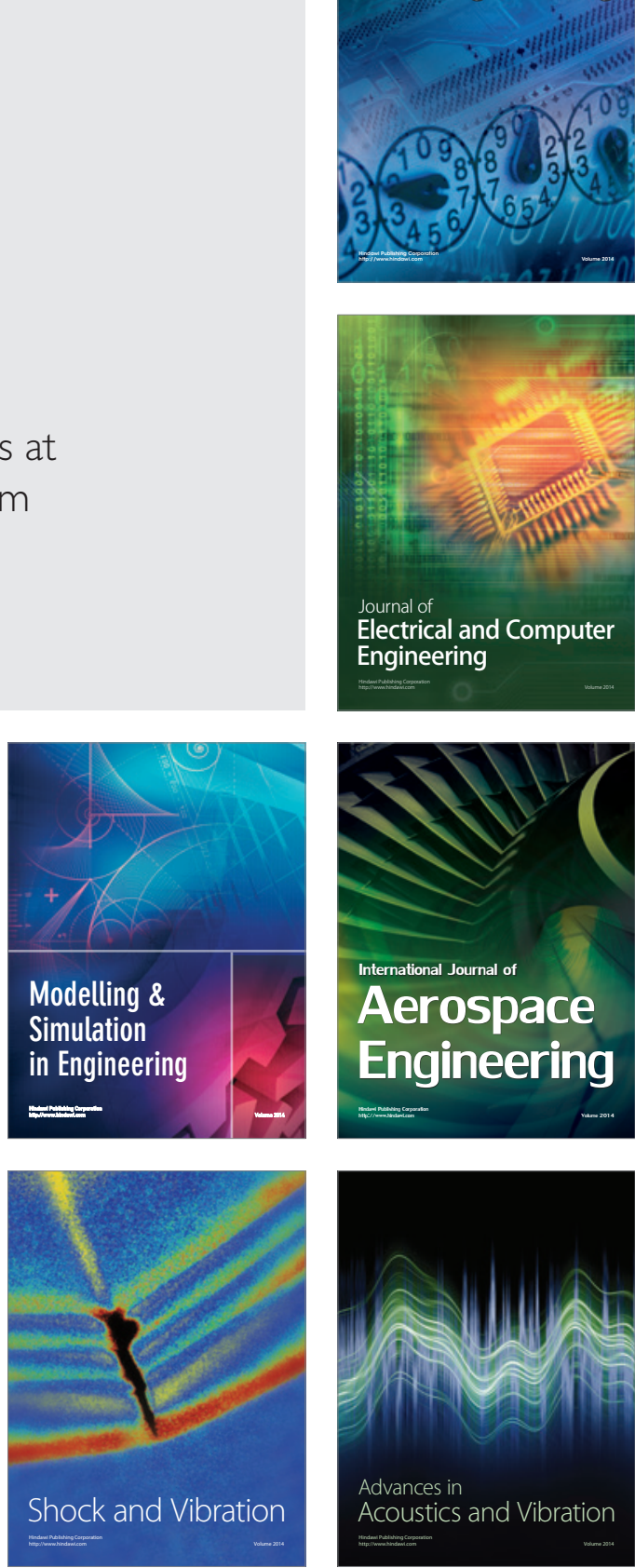